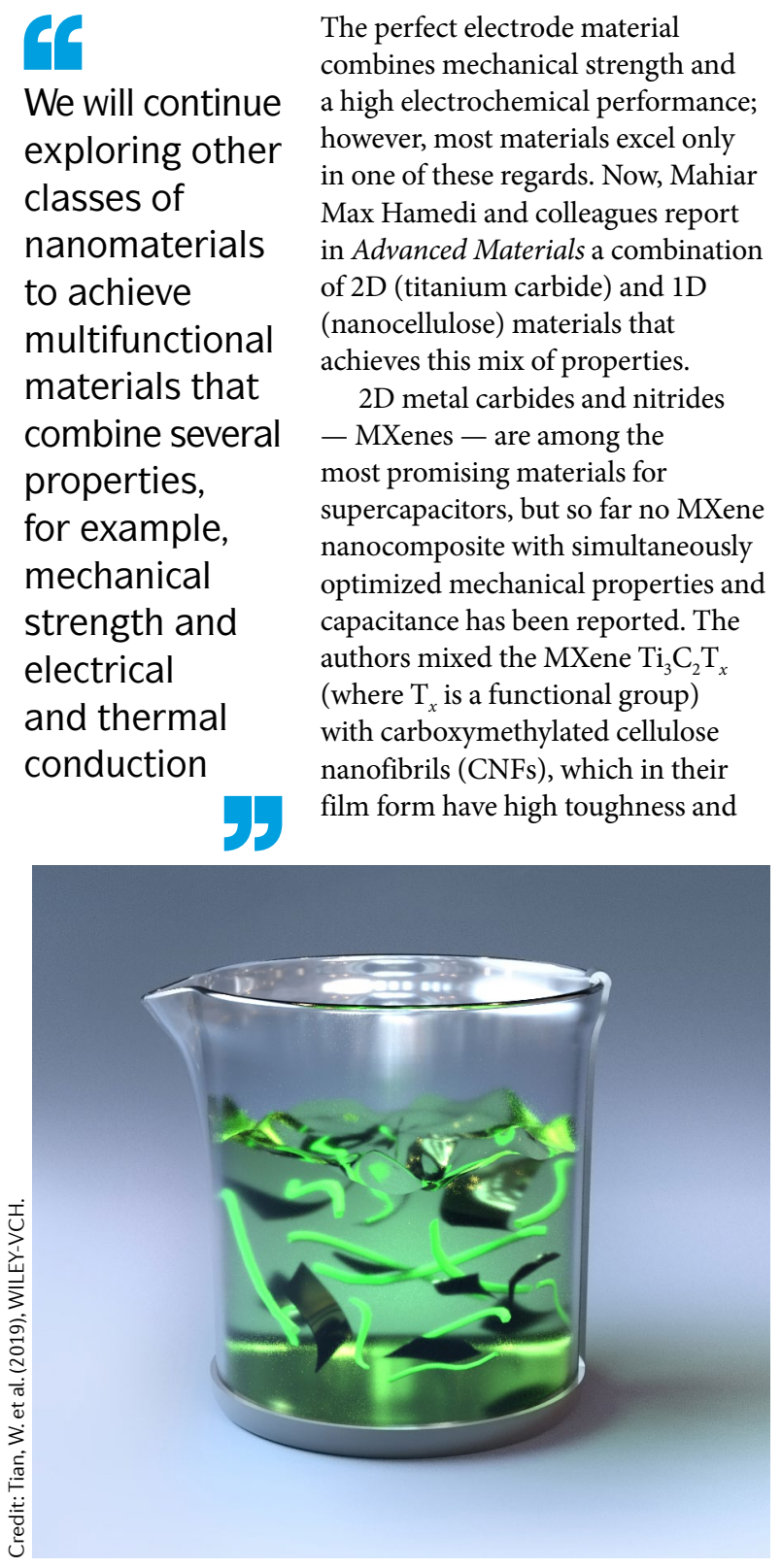

\title{
Superelectrodes for supercapacitors
}

can withstand large strains. Thanks to their high aspect ratio, CNFs can bind MXene flakes to make high-strength materials. "The idea behind this work emerged from our previous use of nanocellulose with carbon nanotubes," explains Hamedi. "We learned that nanocellulose has an interesting interaction with electroactive materials, so we started exploring its combination with $2 \mathrm{D}$ materials."

CNFs and $\mathrm{Ti}_{3} \mathrm{C}_{2} \mathrm{~T}_{x}$ colloidal dispersions with different weight ratios can be made into freestanding hybrid films that have both high electrical conductivity and excellent mechanical strength; their layered structure is similar to that of MXene films. The basis of their attractive properties are the strong interfacial interactions between flakes and fibrils: electrostatic forces cause long-range repulsion, which stabilizes the dispersion, whereas the functional groups on the transition metal layer surface promote adhesion between the two components.

The geometrical proportions between the two types of materials are important, as the long cellulose fibrils bind MXene flakes and lock adjacent layers - indeed cellulose nanocrystals, which are shorter than CNFs, have little effect on the mechanical properties of $\mathrm{Ti}_{3} \mathrm{C}_{2} \mathrm{~T}_{x}$ when they are combined to form a composite material. "We will continue to study how the geometry of different nano-objects affects their structure and properties in composites," says Hamedi. "We have learned that the length difference between rods and sheets has an enormous impact on the strength of the composite they form." Vacuum pressing further improved the strength of the material.

Electrochemical measurements highlighted the high conductivity of the composite, which is probably due to the minimal insulating gaps introduced between MXene flakes by the thin CNFs; vacuum pressing also doesn't significantly lower the capacitance of the MXene flakes. Cyclic measurements revealed a $100 \%$ capacitance retention after 10,000 charging-discharging cycles.

Using the dispersions as inks, the authors printed microsupercapacitors on flexible substrates, in which the $\mathrm{Ti}_{3} \mathrm{C}_{2} \mathrm{~T}_{x}$ I CNF electrodes acted as both the current collector and active material to store electrochemical energy. The performance was comparable to that of pristine MXene microsupercapacitors, but combined with exceptional mechanical properties: the devices could be bent and rolled without performance degradation. "We will continue exploring other classes of nanomaterials to achieve multifunctional materials that combine several properties, for example, mechanical strength and electrical and thermal conduction," concludes Hamedi.

Giulia Pacchioni

ORIGINAL ARTICLE Tian, W. et al. Multifunctional nanocomposites with high strength and capacitance using 2D MXene and 1D nanocellulose. Adv. Mater. https://doi.org/10.1002/adma.201902977 (2019) 